\title{
Philonsorbonne
}

14 | 2020

Année 2019-2020

\section{L'objet de la critique marxienne dans la Contribution à la critique de la philosophie du droit de Hegel}

\section{Valentin LEROY}

\section{(2) OpenEdition}

Journals

Édition électronique

URL : https://journals.openedition.org/philonsorbonne/1406

DOI : 10.4000/philonsorbonne.1406

ISSN : 2270-7336

Éditeur

Publications de la Sorbonne

\section{Édition imprimée}

Date de publication : 14 février 2020

Pagination : 47-75

ISSN : 1255-183X

\section{Référence électronique}

Valentin LEROY, «L'objet de la critique marxienne dans la Contribution à la critique de la philosophie du droit de Hegel », Philonsorbonne [En ligne], 14 | 2020, mis en ligne le 26 février 2020, consulté le 08 juin 2021. URL : http://journals.openedition.org/philonsorbonne/1406 ; DOI : https://doi.org/10.4000/ philonsorbonne.1406 


\title{
L'objet de la critique marxienne dans la Contribution à la critique de la philosophie du droit de Hegel
}

\author{
Valentin LEROY
}

Il s'agit ici de s'interroger sur l'unité et la fonction de ce que l'on entend par critique dans la Contribution à la critique de la philosophie du droit de Hegel de Marx. En effet, si l'on s'accorde sur la cible de cette critique, à savoir la philosophie hégélienne contenue dans les Principes de la philosophie du droit, celle-ci se donne dans plusieurs modalités qui semblent parfois opposées voire contradictoires. On voudrait montrer qu'il y a bien une unité critique sous-jacente sauf qu'elle n'est pas tournée exclusivement vers la destruction des thèses hégéliennes, mais vers une méthode en cours d'élaboration dont l'horizon est la saisie théorique du réel socio-politique, moyennant tout le vague que ces termes convoient nécessairement, et qui est pour ainsi dire constitutif de cette méthode qui se cherche elle-même.

Si le concept de critique accompagne l'itinéraire intellectuel marxien, soit comme titre explicite comme ici, soit comme sous-titre (La Sainte Famille, "critique de la critique critique »), jusqu'au maître-œuvre, Le Capital, "critique de l'économie politique », il n'est pas indifférent de rappeler qu'il s'agisse ici de la première œuvre de l'auteur qui prenne la forme d'une critique - à charge pour nous de comprendre ce que cela peut vouloir dire.

Plus précisément, cette critique consiste en une explication serrée des paragraphes 261 à 313 des Principes de la philosophie du droit de Hegel dans lesquels Hegel expose l'idée d'État au sens strict. Ce passage est luimême contenu à l'intérieur de la section consacrée au droit politique ou public interne, soit les paragraphes 257 à 360, qui forme dans cet ouvrage la troisième section de la vie éthique. Ces 131 feuillets écrits entre mars et août 1843 à Cologne puis à Kreuznach attestent du sérieux qu'il applique à la question politique mais aussi philosophique de la connaissance de cet objet 
conceptuel qu'est l'État. Il semble donc que ce soit au travers d'une révision critique de la relation entre État politique et société civile bourgeoise, telle qu'elle est conçue et énoncée dans les Principes de Hegel, que Marx entame sa carrière de philosophe ou de théoricien politique.

Le texte de Hegel dans sa littéralité fixe bien le cadre réflexif de la pensée marxienne balbutiante. Mais dire cela, c'est déjà soulever une série de questions qui renvoient d'emblée au statut conceptuel de la critique marxienne: soit il s'agit de critiquer de façon externe le texte de Hegel, c'est-à-dire à l'aune de contenus théoriques qui n'appartiennent pas à la conceptualité hégélienne, soit il s'agit à l'inverse d'adopter une critique interne en se plaçant à l'intérieur des thèses adverses pour mieux en relever les incohérences ou difficultés. S'il s'agit de mesurer la cohérence de la doctrine hégélienne, alors Marx veut s'expliquer avec Hegel dans l'intérêt de Hegel. S'il s'agit de mesurer la pertinence de la théorie hégélienne avec la réalité politique, entendue comme description de la réalité politique, alors il s'agit pour Marx de s'expliquer avec Hegel pour autre chose que la sauvegarde ou la juste appréciation du système du maître.

On aurait spontanément envie de choisir la seconde option tant les écrits ultérieurs de Marx et surtout L'Idéologie allemande (dont certains thèmes sont annoncés dans ce texte à travers la reprise partielle de l'anthropologie feuerbachienne) rompent avec l'hégélianisme. Mais ce serait oublier une question qui semble être préalable à toutes celles qui concernent le statut de la critique, à savoir celle de la nécessité d'une telle critique : pourquoi examiner la philosophie politique de Hegel et pas une autre. À ces questions liminaires répond la notion qui donne son titre au livre, la critique, car c'est dans les contenus théoriques résultant de la critique des textes de Hegel que semblent reposer les éclaircissements nécessaires aux problèmes évoqués. À l'inverse, l'examen des différentes formes de critiques approfondit les difficultés; on serait en effet bien en peine de trouver une seule forme de critique, ou une méthode critique mais bien plusieurs critiques ou plusieurs modèles de critique selon l'introduction à l'édition GEME du texte'

On trouve d'une part une critique qui se réclamerait d'un modèle génétique : reconduction de l'État à son sujet véritable, la société civile selon la localisation feuerbachienne d'une inversion du sujet et du prédicat par rapport à la méthode hégélienne. Autrement dit, il s'agirait d'appliquer la méthode de Feuerbach non plus à l'objet religieux mais à l'objet politique. Mais cela soulève d'emblée une question sur la validité de cette éventuelle transposition du politique au religieux : s'il s'agit effectivement d'utiliser Feuerbach en terre politique, alors pourquoi nécessairement en passer par Hegel?

D'autre part, il semble y avoir un modèle empirique car le schème feuerbachien de l'inversion sujet-prédicat ne constituerait qu'un pan du dispositif critique mis en place par Marx contre Hegel. L'État est susceptible

1. K. Marx, Critique de la philosophie du droit de Hegel, Paris, éd. Sociales, 2018. p. 25-30. 
d'être connu scientifiquement non pas parce qu'il s'agit en pure logique hégélienne d'un moment du développement spéculatif du concept, mais parce qu'il est une réalité empirique. Ainsi, en ne considérant que le concept de manière abstraite, Hegel ne penserait pas son sujet mais le raterait, ce qui le conduirait nécessairement à rater derechef la description politique à laquelle il prétend ou voudrait se livrer. Hegel plaquerait ses propres concepts sur l'existant, et ainsi le mystifierait puisqu'il ne l'examinerait pas pour lui-même, mais à travers le prisme déformant de sa propre philosophie. Si la pertinence d'une telle critique est facile à comprendre, sa validité l'est beaucoup moins : d'où peut venir cette critique de Marx ? S'il critique Hegel parce que celui-ci déploie une fausse conceptualité qui n'est pas à même de décrire proprement l'État, c'est que Marx connaît alors cet objet mieux que Hegel, et est capable d'en évaluer les bonnes ou mauvaises descriptions. La connaissance d'un tel objet devenant alors le critère à partir duquel critiquer la conception hégélienne de l'État, cela revient à s'étonner alors d'une absence : si Marx peut connaître scientifiquement l'État, pourquoi n'at-il pas développé cette théorie pour elle-même au lieu de développer une critique de Hegel ? Un tel étonnement s'inscrit dans le sillage du précédent : quelle est la pertinence de la critique de Hegel pour la connaissance, ou la théorie du politique?

Un troisième modèle ou régime ${ }^{2}$ de critique serait dialectique : reconnaître l'effectivité dans l'expérience plutôt que dans le concept et faire valoir une effectivité conceptuelle authentique basée sur elle. Il s'agirait ici cette fois d'un retour, quoique sous une autre forme, au premier modèle en ce que ce régime aurait à charge de critiquer la méthode dialectique de Hegel elle-même. L'État hégélien serait alors la mystification ultime de la logique hégélienne, dont la spéculation fallacieuse culminerait dans le registre de la théorie politique, offrant ainsi le meilleur point d'entrée possible à sa critique, ce que Marx aurait parfaitement vu. D'un projet de connaissance de la politique et de ses objets, on retomberait dans un projet historicophilosophique dans la mesure où il faudrait s'expliquer non pas tant pour soi-même qu'avec Hegel dans l'intérêt de l'hégélianisme. Troisième modèle qui appelle conjointement les soupçons soulevés pour les deux autres : pourquoi critiquer Hegel ? Et de toute façon, comment le critiquer, dans la mesure où cela suppose une méthodologie qui reviendrait à dépasser les difficultés rencontrées par celle de Hegel ?

Ces difficultés afférentes aux régimes disparates de critiques recouvrent les questions que l'on a esquissées : veut-on atteindre Hegel ou le politique (si tant est que l'on puisse définir une telle notion de manière transparente) ? Si l'on veut atteindre Hegel, pourquoi et comment se placer sur le bon site pour le critiquer? Si l'on veut atteindre le politique, pourquoi se placer en territoire hégélien? Si l'on veut s'expliquer avec les deux, pourquoi leur rapport est-il nécessaire dans le procès critique? Les trois modèles

2. Id., p. 29. 
de critique, dans leur rapport à Hegel et dans leur volonté éventuelle mais probable de connaître le politique, nous enjoignent donc de comprendre ce que peut vouloir dire critiquer pour Marx dans cet ouvrage.

On a là trois modèles différents qui posent chacun leurs problèmes que l'édition GEME place cependant sous la houlette d'un seul modèle ou régime : la critique de la spéculation hégélienne ${ }^{3}$. L'unicité postulée de ce modèle revient à trancher a priori une bonne partie des questions soulevées ci-dessus: la critique marxienne, comme du reste le titre de l'ouvrage l'indique, serait avant tout et surtout, pour ne pas dire exclusivement, une explication avec la spéculation hégélienne. Mais les éditeurs reconnaissent, sans pourtant vraiment dénier cette unification a priori des différentes critiques, que « loin de mettre en place une méthode critique unifiée, c'est à une lecture résolument pluraliste du motif de la critique de la spéculation que nous contraint le Manuscrit de Kreuznach. Le texte mobilise de façon généralement peu thématisée différents modèles critiques ${ }^{4}$. Ce que nous apprend cette recension difficile des modèles ou régimes de critique dans ce texte est crucial : les questions que posent la rédaction pour ne pas dire l'existence de ce texte singulier sont les mêmes que celles que soulève l'utilisation mystérieuse de la notion de critique ou des critiques, tant et si bien que se demander ce que cherche à faire Marx dans ce texte revient à éclairer ce moment de son itinéraire intellectuel et ce que veut dire critiquer pour lui.

Jusqu'à présent donc, on comprend qu'il y a plusieurs formes de la critique marxienne à l'œuvre dans ce texte, supposément unifiables dans la destruction théorique de la spéculation hégélienne, ce qui ne va pas sans les difficultés évoquées ci-dessus. L'hypothèse que l'on suivra est différente et permet de désamorcer l'unité critique trop lâche de la cible hégélienne. S'il y a un seul objet de la critique marxienne, ce n'est pas la spéculation hégélienne, mais le réel politique qui doit par elle s'interposer et servir de critère pour évaluer en retour la théorie de Hegel. C'est face à ce réel politique - l'état que la théorie de Hegel entend décrire ou conceptualiser spéculativement par exemple - que la cohérence du système pourra être évaluée.

Mais c'est aussi en prenant en compte cet unique objet - le réel politique - que l'on peut expliquer l'unicité du projet critique marxien autant que le choix d'une explication pour soi avec Hegel. Nous disons bien avec Hegel, car la logique spéculative des Principes de la philosophie du droit en ce qu'elle a d'abstrait - au sens marxien du texte - fait le plus mal à ce réel; et c'est une explication pour soi car il s'agit de dessiner en creux, dans la silhouette encore mystérieuse de ce réel, une autre méthode : non pas partir du concept et de son auto-détermination, mais du réel en tant que la théorie a d'abord à le connaître et non à lui faire violence. Se joue donc

3. Ibid., p. 17-19.

4. Ibid., p. 30 . 
peut-être une autre conception de la théorie et de la critique, non plus une critique qui aurait à détruire les énoncés adverses à l'aune de normes externes ou des siennes propres, non plus une critique spéculative, mais une critique qui cherche à connaître le réel pour lui-même en évaluant une théorie concurrente et dont l'autorité avérée doit être contestée.

Mais dire cela, c'est se placer en bout de course, et par là, fort du regard rétrospectif triomphant, effacer toutes les difficultés que l'on a énumérées plus haut. C'est en effet faire comme si Marx disposait déjà de ce critère à l'aune duquel mesurer toute théorie politique, notamment la plus conceptuelle et abstraite sans doute à ses yeux. Or, outre le fait que le réel politique est un concept tout à fait indéterminé et qui pose les problèmes que nous verrons, il faut faire droit à l'aspect processuel et agonistique de la pensée marxienne : Hegel pense contre et c'est en pensant de cette manière que les concepts qui lui permettent de penser (contre, donc) se forment.

Si l'on a utilisé les termes «modèle », « forme » ou même « régime» de critique, il faut présupposer que la critique est avant tout fonction et fonction théorique ; c'est en la faisant fonctionner sur ce qu'elle a à critiquer qu'elle livre de nouveaux objets théoriques. Marx, dans ce texte (nous en faisons l'hypothèse) acquiert cet étalon-mesure ad hoc de la théorie politique hégélienne, le réel politique, par lequel il est à même de critiquer Hegel. Que veut-on dire par "réel politique »? Rien de moins que ce qui appartient au politique et au social et qui est d'abord et d'emblée étranger à la théorie. Ainsi, lorsque Marx entend examiner le paragraphe 261 des Principes de la philosophie du droit par lequel notre ouvrage débute ${ }^{5}$, il appuie l'idée que l'État serait une «nécessité extérieure » à ce qu'il surplombe dans la société, soit dans les mots de Hegel « les sphères du droit privé et du bien être privé, de la famille et de la société bourgeoise $»^{6}$. Mais pour savoir à quoi l'État est extérieur, il faut comprendre ce que désignent ces concepts de "société civile bourgeoise » ou de "famille », alors même que ces concepts portent une charge théorique hégélienne - celle-là même qu'il s'agit d'évaluer ; autrement dit, ces concepts sont à la fois le point de mire et l'écran nécessaires à la saisie théorique de ce qu'ils prétendent désigner, ce qui signifie par là-même que c'est le réel derrière ceux-ci qu'il s'agit d'atteindre. D'une certaine manière, la critique marxienne doit réussir l'opération impossible de sauter au-dessus de ces concepts pour atteindre ce qui ne peut apparaître sans eux.

C'est pourquoi en ce sens le réel politique est à la fois le terme et le point de départ de la critique marxienne. Paradoxe, mais seul point de vue permettant à la fois d'unifier le projet critique, et le «pluralisme» de ses différents modèles ou régimes fonctionnels. D'où peut-être aussi, finalement, l'embarras de qui voudrait discriminer exhaustivement les différents

5. Ibid., p. 77.

6. Ibid., p. 77 ou F. Hegel, Les Principes de la philosophie du droit, trad. J.-F. Kervégan, Paris, Presses Universitaires de France, 1998, p. 432-434. 
modèles de critique en les reconduisant à un projet aussi précis que la critique de la spéculation hégélienne. L'objection que l'on pourrait opposer ici est qu'un critère aussi indéterminé que la simple mention du réel politique ne peut emmener une critique bien loin ; mais c'est précisément la raison pour laquelle le texte porte les nombreuses traces des gesticulations de Marx avec son propre discours et non seulement avec le texte hégélien. Il s'agit simplement de prendre au sérieux l'idée d'une explication théorique pour soi, c'est-à-dire jamais assurée ni de sa méthode, ni de ses résultats, d'où cette indétermination et d'où sans doute aussi l'abandon de cette notion dans l'itinéraire intellectuel ultérieur de Marx.

\section{Le droit comme passage obligé d'une telle critique}

Une première manière de répondre aux questions que suscitent la fonction critique dans ce texte est de s'intéresser non pas d'emblée à Hegel pour lui-même, mais à ce qu'il veut mettre en place dans les Principes, c'est-à-dire une théorie spéculative du droit. Si l'on suppose que la critique marxienne s'attache détruire la spéculation hégélienne, on regarde exclusivement du côté du déploiement spéculatif des concepts, qui ressortit somme toute à la plupart des textes hégéliens - cependant, une telle formulation tend à oublier qu'il s'agit pour Marx de critiquer la philosophie $\mathrm{du}$ droit. N'est-ce pas précisément ce qui confirme notre hypothèse ? S'il s'agit d'atteindre le réel politique, pour l'heure complètement indéterminé, par l'intermédiaire des thèses hégéliennes, alors le droit semble être le meilleur point d'entrée. D'une part, le droit est bien une émanation théorique censée régir des rapports sociaux véritables car non théoriques, et d'autre part, il ne s'agit pas d'un concept intégralement produit par Hegel mais pour ainsi dire trouvé là. L'hypothèse se précise donc: atteindre le réel politique justifie et nécessite d'en passer par une critique du droit. Or pour le jeune Marx, cela veut dire essayer d'appréhender une théorie du droit qui puisse elle-même prétendre approcher au plus près ce réel, ce que font les Principes de Hegel.

Mais la question générale du droit dans l'œuvre de Marx est difficile à réunir en un tout cohérent et unifié. Pire, la question du droit semble se rattacher à des thèses marxiennes primordiales et bien connues sans que sa spécificité soit examinée, conduisant ainsi parfois à des idées contradictoires mais qui semblent pourtant cruciales. Le droit fait ainsi partie de la « superstructure » dans la fameuse préface de la Contribution à la critique de l'économie politique, c'est-à-dire des constructions idéologiques qui s'édifient sur la base matérielle des rapports de production et qui ont à charge de la justifier. Dans la Question Juive, où le thème du droit et notamment des droits de l'homme est étudié le plus à fond et le plus pour lui-même, Marx semble en effet porter des analyses similaires puisque les droits de l'homme sont reconduits, en tant que droits exclusivement formels, à la justification indue d'intérêts spécifiques de classe, dont l'accession à la propriété. 
La question est sans doute difficile et nous ne nous n'y intéresserons pas pour elle-même mais pour ce qu'elle met en jeu et au jour concernant la critique marxienne dans la Contribution à la critique de la philosophie $d u$ droit de Hegel. Si le droit peut être considéré à un stade tardif de l'œuvre de Marx comme étant d'une part un pur produit idéologique et de l'autre l'expression de rapports matériels, c'est qu'il se situe sans doute entre ces deux sphères, entre l'idéologique et l'expression fidèle des rapports sociaux qu'il doit régler et pacifier. Un passage de l'autodéfense de Marx dans le procès contre le Rheinischer Kreisausschuß der Demokraten, publié dans la Neue Rheinische Zei-tung du 25 février 1849, nous met sur une voie plus précise : «la société ne repose pas sur la loi. C'est une illusion de juristes. C'est au contraire la loi qui repose sur la société, qui doit être l'expression de ses intérêts et des besoins communs issus chaque fois du mode de production matériel contre l'arbitraire individuel $»^{7}$. On comprend donc que le droit serait au départ comme porteur d'une certaine forme de neutralité axiologique et idéologique, ce qui ne l'empêcherait pas par la suite d'être récupéré par la bourgeoisie pour justifier ses intérêts. En tout cas, ce qui est plus intéressant pour nous, c'est précisément cette difficulté : le droit n'est pas seulement idéologique, mais est peut-être aussi le système théorique qui exprime des rapports pratiques. Ainsi se trouve-t-il déjà à la confluence de l'idéel et du matériel, du théorique et du pratique, sans être totalement l'un ni totalement l'autre. Un texte central de la Critique de la philosophie du droit semble aller dans le même sens :

Hegel caractérise le droit privé comme le droit de la personnalité abstraite ou le droit abstrait. Et en vérité, il le faut développer comme l'abstraction du droit et, partant, comme le droit illusoire de la personnalité abstraite, de même que la morale telle que la développe Hegel est l'existence illusoire de la subjectivité abstraite. Hegel développe le droit privé et la morale comme des abstractions de ce genre, de quoi il ne suit pourtant pas, chez lui, que l'État, l'éthicité, qui les a pour présuppositions, ne puisse être rien d'autre que la société (la vie sociale) de ces illusions, mais à l'inverse, la conclusion qui en est tirée est qu'ils sont des moments subalternes de cette vie éthique. Mais qu'est-ce que le droit privé sinon le droit, qu'est-ce que la morale sinon la morale de ces sujets de l'Etat ? ${ }^{8}$

Qu'est-ce à dire ici ? Le nerf de la critique marxienne consiste à soupçonner et à questionner la théorie hégélienne du droit, de manière générale et précise à la fois : Hegel théorise le droit à partir d'une abstraction et semble-t-il avec cohérence car ce n'est pas le contenu d'une telle théorie qui est proprement contesté mais le fait que le droit peut être et devrait être l'émanation de rapports sociaux concrets. D'une part, le droit semble donc

7. K. Marx, «Der Prozeß gegen den Rheinischen Kreisausschuß der Demokraten », in K. Marx and F. Engels, Werke, band 6, Berlin, Dietz Verlag, 1959, p. 245 (cité par S. Petrucciani, "Les multiples dimensions de la critique marxienne du droit», Droit et Philosophie, 10, (2018), p. 15.

8. K. Marx, Critique de la philosophie du droit de Hegel, Paris, éd. Sociales, 2018, p. 207. 
bien être idéologique comme la simple expression théorique ou idéelle de rapports matériels, ou encore le reflet conceptuel de ces rapports. Pourtant, d'autre part, ce texte ajoute un point crucial : "Hegel développe le droit privé et la morale comme des abstractions de ce genre, de quoi il ne suit pourtant pas, chez lui, que l'État, l'éthicité, qui les a pour présuppositions, ne puisse être rien d'autre que la société (la vie sociale) de ces illusions »: le droit n'est pas n'importe quel rapport intellectuel mais précisément celui qui doit régir tous les autres rapports sociaux, c'est-à-dire aussi la structure et le fonctionnement de la société. Il s'agit donc pour Marx d'avoir le meilleur point d'entrée pour penser la société, qui se trouve dans l'analyse du droit : critiquer une théorie du droit c'est se donner les moyens de penser la société dans son ensemble non pas ex nihilo mais d'emblée à l'articulation de l'idéel et du matériel.

On pourrait ici faire une objection majeure: idéel/matériel, superstructure/infrastructure, idéologie/ matière sont des couples conceptuels du marxisme non encore élaborés à l'époque du texte qui nous intéresse, des outils dont se dotera Marx une fois qu'il voudra critiquer et la métaphysique allemande (l'Idéologie Allemande) et plus tard l'économie politique (Contribution à la critique de l'économie politique). Précisément, ce que nous voulons montrer c'est que toutes ces oppositions traditionnelles du marxisme vont chercher à s'élaborer dans ce texte à l'état nécessairement inchoatif, ou plutôt comme projet qui tend vers ce que cherchent à exprimer ces concepts ; une connaissance du social et du politique qui puisse échapper au prisme déformateur des intérêts d'une classe en particulier naît à l'occasion de la critique de ce texte de Hegel. Ce projet se porte donc sur certaine critique, celle de la Contribution à la critique de la philosophie du droit de Hegel ; autrement dit en critiquant Hegel, Marx cherche une théorie à même de connaître le réel. Est-ce un hasard si quelques pages avant le

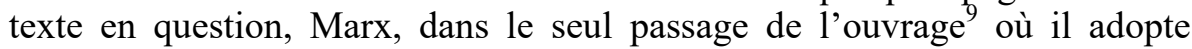
un point de vue réflexif sur sa méthode ou plus prudemment sur sa critique, se charge de la penser pour elle-même dans ses finalités théoriques?

Selon Marx, la critique vulgaire fait erreur en ce qu'elle propose une solution dogmatique à ces problèmes théoriques :

il s'agit là même encore d'une critique dogmatique, qui lutte avec son objet tout comme on a jadis en quelque façon liquidé le dogme de la sainte trinité par la contradiction de 1 et 3 . La vraie critique, en revanche, montre la genèse interne de la sainte trinité dans le cerveau humain. Elle décrit son acte de naissance. Ainsi la critique véritablement philosophique de la constitution actuelle de l'État ne s'arrête pas à mettre en lumière des contradictions en tant qu'elles existent, elle les explique, elle conçoit leur genèse, leur nécessité ${ }^{10}$.

9. Et un des seuls passages de l'œuvre de Marx, car les moments où il thématise la notion de critique pour elle-même sont relativement rares au regard de l'importance affichée d'un tel concept pour son œuvre théorique totale.

10. Ibid., p. 187-188. 
La critique dogmatique se contente de repérer les contradictions, ici de l'État, et Marx vise sans conteste Hegel qui localise la contradiction entre État et société civile, ainsi que les contradictions au sein de la société civile mais sans écrire leur genèse. Certes, on comprend l'opposition que Marx trace entre la « vraie critique » et la « critique dogmatique » qui ne fait pas la genèse de son objet pour le comprendre, mais le propos reste assez flou, notamment concernant le relevé de la difficulté dans laquelle se meut la critique dogmatique qui ne peut que «lutter avec son objet ». Car on ne peut comprendre cette lutte qu'à la lumière de l'ébauche de la méthode de la vraie critique qui connaîtrait son objet en en traçant la nécessité interne Mais ce n'est précisément pas ce que semble faire Marx en dehors d'esquisses de passages historiques ${ }^{11}$.

En fait, comme on l'a dit, l'objectif d'une telle entreprise semble être de préciser ce que doit être la «vraie» critique. Si la «vraie» démocratie appelée de nombreuses fois au cours du texte n'a de vraie que la norme épistémique et idéale que Marx veut bien lui conférer, il faut de même mettre au jour le contenu normatif que Marx désigne lorsqu'il mentionne la « vraie critique ». Il semble bien qu'il s'agisse d'une critique qui s'attache à découvrir et connaître son objet sans lui faire violence, sans engager de lutte avec lui mais en en épousant les contours de façon à le connaître tel qu'il est. Question philosophique classique mais qui se pose en des termes différents dès lors qu'il s'agit de connaître la société ; la connaissance de celle-ci présuppose en effet des concepts qui lui soient adéquats, or comment mesurer cette adéquation? C'est bien de cela qu'il est question puisque Marx reproche constamment à Hegel de forcer l'objet à connaître pour le faire entrer même déformé dans l'image que désignent ses concepts. Ainsi, dans le texte précédemment cité, Marx passe subrepticement de la distinction entre "vraie critique » et « critique dogmatique » à une autre appréhension théorique qu'il nomme un "concevoir»: "Mais ce concevoir ne consiste pas comme Hegel le pense à reconnaître partout les déterminations du concept logique, il consiste au contraire à saisir la logique propre de l'objet propre $»{ }^{12}$. Ce « concevoir » assume donc la fonction de la « vraie critique » et se définit pour l'instant sous la plume de Marx, en opposition avec le discours spéculatif hégélien. De même, dans le passage cité à propos de la conception marxienne du droit ${ }^{13}$, non seulement Marx fait mention de la véritable nature de l'éthicité hégélienne qui n'est rien d'autre que la «vie sociale », mais il lui est reconnaissant d'avoir placé la morale sur son socle, l'État : «À de nombreuses reprises, on a attaqué Hegel sur la manière dont il développe la morale. Il n'a rien fait d'autre que développer la morale de l'État moderne et du droit privé moderne. On a voulu séparer davantage la

11. Ibid., p. 213-214, où Marx esquisse une genèse du bicamérisme britannique afin de critiquer la députation issue de la société civile bourgeoise telle que la décrit Hegel au $§ 307$ des Principes de la philosophie du droit.

12. Ibid., p. 188.

13. Cf. supra., p. 5, et op. cit., p. 207-208. 
morale de l'État, l'émanciper davantage ! Qu'a-t-on prouvé par là ? Que la séparation de l'État actuel et de la morale est morale, que la morale n'a rien d'étatique et que l'État n'est pas moral. C'est bien plutôt le grand mérite de Hegel, quoique par un certain côté inconscient (et ceci précisément parce que Hegel a fait passer l'État pour l'idée réelle de l'éthicité), que d'avoir assigné à la morale moderne sa véritable position ${ }^{14}$. On ne peut comprendre ces passages qu'en les lisant conjointement: si la spéculation hégélienne n'est pas critiquée ici et si Hegel a raison sur le rapport entre la morale et l'État, c'est que la morale comme le droit ne flotte pas au-dessus des individus mais est l'expression de certains rapports à l'intérieur de la société. En liant morale et État, Hegel a raison car il rattache une réalité théorique aux rapports pratiques - réels - qu'elle est censée exprimer, « quoique, par un certain côté, inconscient» parce que Hegel lie ces réalités politiques et sociales non à leur développement propre mais à celui logique de l'idée et tend donc à escamoter le réel qu'il prétend pourtant connaître.

À l'inverse, seul un mode de connaissance qui ressortirait à une véritable critique semble capable de mettre au jour le réel politique en expliquant ces contradictions, à l'opposé de toutes les autres pensées critiques. Mais, on voit que cette véritable critique reste encore bien peu déterminée et que Marx ne l'a pratiquement pas mise en œuvre jusque-là.

Ainsi, d'une part le droit a un statut privilégié puisqu'il se situe entre les idées et la matière, entre le théorique et le pratique, et c'est pourquoi Marx choisit cet objet pour amorcer une pensée du social et du politique. D'autre part, cet objet est la présupposition de la critique marxienne dans la mesure où la critique se cherche au contact du texte hégélien. La critique marxienne dépasse le simple cadre d'une révision de la théorie hégélienne du droit mais s'y intéresse tout particulièrement parce que cet objet cristallise ce qu'elle cherche à saisir théoriquement. C'est pourquoi aussi il est possible de dégager les mêmes préoccupations théoriques dans les moments où il ne s'agit plus de s'intéresser au droit pour lui-même.

\section{La critique à la recherche du réel}

Puisque le texte s'attache à suivre les paragraphes des Principes de la philosophie du droit et les commente au fur et à mesure, on ne reprendra pas chaque critique particulière mais on tentera de sélectionner les passages qui peuvent nous aider à comprendre ce qu'est en propre l'objet commun de ces critiques, et donc confirmer les analyses que nous venons d'avancer.

On ne veut pas étudier ce texte pour ses thèses mais pour ce qui s'y joue, et cela revient à prendre au sérieux la tonalité critique de l'ouvrage et à essayer précisément de comprendre ce que veut dire "critique » ici. On ne fera pas comme les commentateurs qui choisissent d'investir à fond cet 
angle quitte à faire du texte une explication exclusive de Marx avec Hegel allant jusqu'à une révision de sa Science de la Logique ${ }^{15}$ - ou qui à l'inverse décident d'oublier cet aspect, pour en faire une collection certes précoce mais néanmoins solide de thèses philosophiques d'un jeune auteur promis à un bel avenir dont il faut alors évaluer la pertinence ${ }^{16}$.

Au début de l'ouvrage, il s'agit pour le Hegel des paragraphes 261 à 271 d'étudier le droit étatique interne, ce que nous appellerions le droit public interne qui doit montrer que l'État est l'effectivité de la liberté, notamment au travers de ses institutions, avant d'en venir plus précisément à celles-ci et à leur organisation constitutionnelle. Les Principes n'étaient censés être qu'un manuel à l'adresse des étudiants de Hegel destiné à soutenir les cours magistraux du professeur. Mais pour Hegel, la philosophie du droit n'est pas anodine car elle doit être l'expression intégrale de l'idée du droit ${ }^{17}$, c'est-àdire l'inscription à même le réel et en son sein du concept spéculatif du droit ; mais ce terme est lui-même préalablement investi de la théorie hégélienne : il s'agit du concept de l'esprit libre dont il fut montré à la fin du parcours de la «philosophie de l'esprit subjectif» qu'il doit précisément se rendre objectif, c'est-à-dire se donner une effectivité juridique et éthique, puis religieuse et scientifique ${ }^{18}$. Pour Hegel, et c'est ce qui fait aussi sa nouveauté en la matière, l'identification de la volonté et de la pensée est totale, et le droit n'est lui-même que l'expression de la volonté à un niveau non individuel. Autrement dit, ce que veut faire Hegel dans ce texte est de fonder les droits et devoirs dans la liberté. L'Etat hégélien est rationnel parce que spéculatif, mais la proposition peut et doit se renverser.

Il s'agit donc du point de départ pour Hegel de la théorie de l'État devant réconcilier la société civile et la famille, et de la critique de Marx puisque l'un des thèmes généraux de la critique marxienne est de contester l'objectivité que représenteraient société civile et famille en tant que moments objectifs du déploiement de l'idée : au contraire, famille et société civile sont les rapports concrets dont procède l'État. Cette thèse, qui est la plus connue du texte par les commentateurs et à laquelle on réduit souvent la fonction critique marxienne, arrive d'entrée de jeu, dès les premières analyses. Mais n'assiste-on pas ici à l'élaboration initiale de cette fonction critique précise, à la recherche du réel comme instrument et norme de la critique, plutôt qu'à celle de ce thème général qui n'apparaît pourtant pas à chaque commentaire de paragraphe ?

15. S. Mercier-Josa, Entre Hegel et Marx : Points cruciaux de la philosophie hégélienne du droit, Paris, L'Harmattan, 1999.

16. M. Abensour, La Démocratie contre l'État, Paris, Le Félin, 2012. Voir également pour un exemple plus récent l'article de S. Haber qui critique pourtant ce dernier : " La critique de l'étatisme hégélien chez le jeune Marx », Droit et Philosophie, 10, (2018).

17. F. Hegel, Les Principes de la philosophie du droit, trad. J.-F. Kervégan, Paris, Presses Universitaires de France, 1998, § 1.

18. F. Hegel, Encyclopédie des sciences philosophiques, trad. B. Bourgeois, Paris, Vrin, 2012, $\S 482$. 
Marx annonce en effet dès le départ que, contrairement à ce que dit Hegel, l'État ne peut être la réconciliation des sphères précédentes qu'abstraitement. Pour Hegel, l'État travaille souterrainement la société civile et la famille en ce qu'elles s'acheminent vers lui dans le temps, mais aussi dans l'effectivité de leur concept. La société civile et la famille ont l'État pour horizon. Or c'est précisément l'inverse pour Marx : la société civile et la famille sont des rapports sociaux qui ne peuvent que déstabiliser l'État, l'État s'édifie sur elles, non le contraire ${ }^{19}$; si l'État peut apparaître comme étant au-dessus de ces sphères ce n'est qu'abstraitement ${ }^{20}$.

Cette thèse est tout à fait frontale et a pour elle l'avantage de faire appel à un argument de bon sens dont l'efficacité serait d'autant plus remarquable qu'il semble procéder d'une observation sans faille : ce que l'on voit dans la société, c'est bien que les rapports économiques (la société civile) et d'organisation sociale immédiate (la famille) sont premiers par rapport à l'État; or comment Marx peut-il savoir cela ? D'où tire-t-il cette thèse à vocation critique ? Une telle critique semble présupposer soit un constat sociologique qui ne peut faire l'économie d'une enquête, soit une théorie politique achevée qui ne peut être que le résultat d'une telle révision critique, ou les deux à la fois. C'est en effet l'observation pétrie de théorie qu'offre la sociologie, ou une mesure critique fournie au préalable par une théorie politique achevée qui peut lui révéler de façon scientifique que la société civile et la famille n'ont rien de commun avec l'État, en sorte que celui-ci ne peut les réunir dans leur effectivité. Si une telle affirmation ne paraît pas étonnante, c'est qu'elle semble s'ancrer dans une observation rétrospective qui nous est commune : les rapports commerciaux, économiques et sociaux (échangistes dira plus tard Marx) sont en effet les véritables fondations de l'État moderne (libéral) dont les constitutions garantissent la tenue mais sans jamais en émaner. L'État se superpose à la société civile mais ne la transforme pas, de même que la société civile ne se métamorphose pas naturellement en État moderne. Le lien spéculatif que la conceptualité hégélienne mobilise n'est en rien attesté par la réalité observée, de sorte que celle-ci paraît faire violence au réel mais dans la mesure où selon nous Marx cherche une méthode concurrente à celle de Hegel pour connaître le réel, ses thèses - dès lors résolument critiques envers Hegel - ne sont pas plus justifiées.

19. «C'est précisément parce que la subordination et la dépendance sont des rapports externes, des rapports qui contraignent l'être autonome et finissent par aller à son encontre que le rapport de la famille et de la société civile bourgeoise est celui de la nécessité extérieure, une nécessité qui s'attaque à l'essence intérieure de la chose ». [...] «La société civile bourgeoise et la famille sans leur développement véritable - c'est-à-dire leur développement autonome et complet, sont en tant que sphère particulière présupposées par l'État », cf. op. cit., p. 81.

20. Marx critique l'effectif ou la fausse effectivité de la médiation : « ce qui fait l'esprit de l'empirie habituelle, ce n'est pas son propre esprit, mais un esprit étranger ». " La famille et la société civile bourgeoise sont les présuppositions de l'État; ce sont elles qui sont à proprement parler actives ; mais dans la spéculation c'est tout l'inverse », id. 
Mais une fois de plus, si ce reproche ne paraît pas extrêmement faible, c'est que nous avons l'intuition de sa pertinence, mais une intuition et un concept aussi indéterminé que celui de réel ne font pas une critique acerbe. Et c'est précisément ce que semble également penser Marx, puisque sans aucune transition, il nous propose un autre type de critique : au lieu de se placer du point de vue extérieur, pour juger la théorie à l'aune de la réalité (fût-elle scrutable de façon transparente), au lieu donc de continuer sur le mode d'une critique externe, Marx adopte une forme interne: se placer à l'intérieur de la théorie ciblée pour en viser les incohérences ou en soulever les problèmes :

La répartition du matériau de l'État à même l'individu singulier à travers les circonstances, l'arbitre et le choix que chacun fait de sa vocation n'est pas exprimée purement et simplement comme s'il s'agissait de ce qui est véritable, de ce qui est nécessaire ou de ce qui est justifié en et pour soi ; ils ne sont pas donnés en tant que tel pour le rationnel ; mais ils le redeviennent pourtant d'un autre côté du simple fait qu'ils sont donnés pur une médiation apparente... ${ }^{21}$

Marx se tourne donc derechef vers la théorie hégélienne (d'où l'idée de médiation); fort du rapport réel inscrutable, il appuie sur l'idée que ce rapport réel n'a rien d'intégralement rationnel, comme le voudrait pourtant la théorie hégélienne spéculative : c'est précisément parce que ces sphères y sont intégrées de force qu'elles peuvent après coup devenir apparemment rationnelles, ce qui veut dire que le système va leur transférer un ersatz de rationalité, qui de toute façon ne leur appartient pas en propre et ne peut suffire à la définir à plein.

Que se passe-t-il donc dans ce mouvement pendulaire entre critique externe faible et critique interne qui s'en alimente ? C'est bien à travers l'extériorité du réel que Marx peut critiquer de manière interne la théorie hégélienne, et en même temps, comme on le voit, cette critique ne peut se dire pour l'instant que par l'intermédiaire des catégories hégéliennes, dans son vocabulaire même : société civile, famille, État, médiation. Plus donc qu'une fonction critique qui s'appuie sur ce qu'elle cherche à mettre en évidence même, c'est un discours critique qui peine à se dire lui-même faute d'une conceptualité adéquate. D'où le caractère répétitif de cette fonction critique qui cherche toujours à isoler son objet en formulant les mêmes reproches à ce qu'elle critique - tentative condamnée à ne pas réussir complètement. C'est pourquoi cette fonction critique doit osciller entre diverses formes: interne, puis externe sans jamais s'y réduire car peu importe la méthode utilisée pour déstabiliser la thèse adverse, l'enjeu est ailleurs : dans ce que cette critique n'arrive pas encore à dire et circonscrire. Ainsi lit-on un peu plus loin: «La fin de leur existence n'est pas leur existence elle-même, au contraire l'idée détache d'elle-même ces 
présuppositions $\gg{ }^{22}$. Il s'agit de montrer dans le droit fil de ce qui précède que la théorie spéculative n'a qu'un seul but, ne jamais penser ces réalités pour elles-mêmes mais pour les intégrer au système. Marx montre « ce qu'il y a derrière le rideau $\gg{ }^{23}$ comme étant tendanciellement artificiel, c'est-à-dire faisant violence à la nature du réel ${ }^{24}$.

Et dans les pages suivantes, c'est à nouveau une critique interne de la théorie hégélienne qui semble être mise en branle. Quel est le problème ? C'est que le lien entre société civile, famille d'un côté et État de l'autre n'est pas clair: le passage n'est pas déduit de l'essence particulière de la famille ni de celle de l'État mais du rapport universel de la nécessité et de la liberté $^{25}$. Pour Marx, on trouve artificiellement une détermination abstraite pour médier deux réalités concrètes. Il s'agit donc bien d'une critique interne, mais qui dénonce l'abstraction d'une théorie pour mieux porter son soupçon: si elle fonctionne par abstraction, celle-ci risque d'escamoter les particularités réelles des sphères examinées - et encore une fois, ces particularités ne sont jamais nommées pour elles-mêmes, faute de les connaître. C'est pourquoi Marx poursuit avec une critique au départ d'apparence externe du paragraphe 269 : « on est parti de l'idée abstraite, celle du développement au sein de l'État, [...] il ne s'agit pas de l'idée politique, mais de l'idée abstraite dans l'élément politique », ce qui l'amène une fois de plus à une critique interne, en tous points similaire à la précédente: Hegel veut retrouver l'idée partout et Marx fustige son abstraction $^{26}$.

Ce que l'on constate, c'est bien la trame d'une séquence qui a l'air plus unifiée ce que l'on veut bien dire habituellement sur la critique marxienne. Certes, nous n'avons pas toujours tranché la question de savoir pourquoi Marx s'attache précisément au texte de Hegel, étant donné que ces considérations brèves et initiales nous montrent plutôt ce qu'il critique et non pourquoi une telle critique. Cependant, nous comprenons au moins maintenant ce que fait Marx : il y a bien un va-et-vient des formes de la critique, entre une attaque qui semble s'alimenter d'une intuition, elle-même

22. Op. cit., p. 82, ou encore : "l'idée effective ne s'abaisse à la finité de la famille et de la société civile bourgeoise que pour les relever et, par là, produire son infinité et en jouir », idem, p. 81-82.

23. Ibid.

24. Op. cit., p. 83 : «l'état de fait d'où l'on part n'est pas saisi en tant que tel, mais en tant que résultat mystique $»$.

25. Op. cit., p. 85.

26. Op. cit., p. 86 : « il reste des différences qui ne sont pas saisies conceptuellement » et p. 90 : « ce n'est pas de l'organisme que l'on part mais de l'idée de l'État ». Marx repart donc de l'idée abstraite pour pointer ses défauts : elle ne pense pas ce qu'elle veut penser, mais sert plutôt de position nécessaire dans un système théorique qui devrait penser la politique mais qui est plus occupé à se penser lui-même, et n'atteint donc que l'abstraction au moment même où le sujet devrait être le plus concret, « ce qu'il fait ce n'est pas développer sa pensée à partir de l'objet en suivant une manière de penser qui a déjà mis fin à sa course et dont sa course s'est achevée à l'intérieur de la sphère abstraite de la logique ». 
ancrée dans un « réel $^{27} »$ invisible et hors du texte puisque Marx n'en parle jamais ; c'est celle-ci qui va déclencher la critique interne et externe de la théorie de Hegel. Ces deux types de critique se soutiennent mutuellement: c'est parce que le réel n'est pas tel que le décrit Hegel que sa théorie présente des difficultés en son sein, mais c'est aussi parce que sa théorie n'est pas complètement achevée qu'elle ne peut décrire adéquatement le réel. Ce qui se passe dans les premières pages du texte nous semble décisif pour ce qui nous concerne : rien moins que la mise en place d'un élan critique tel que décrit plus haut; ce qui veut dire aussi en creux que Marx, au contact de Hegel, n'a qu'un reproche à lui faire - mais un reproche décisif : sa théorie est incapable de saisir la pratique, le concret, ou le réel. C'est bien ce que semble confirmer la fin de notre passage où Marx, emporté par cet élan, propose une remarque critique qui relève autant de la critique interne que de la suggestion : il aurait fallu prendre pour point de départ l'esprit effectif, car alors on aurait développé réellement et matériellement celui-ci, on lui aurait donné un contenu concret: "si l'on avait pris pour point de départ l'esprit effectif alors la fin universelle était son contenu, les différents pouvoirs sa manière de s'effectuer, son existence réelle ou matérielle ; la déterminité de ces derniers aurait précisément été à développer en partant de la nature de sa fin ${ }^{28}$. C'est bien encore une fois cette réalité socio-politique qui sert de mesure et qui le doit sous peine de travestir le réel dans la logique comme le fait Hegel : «ce n'est pas la philosophie du droit mais bien la logique qui constitue le véritable intérêt ${ }^{29}$. Mais comme on l'a vu, ce reproche peut à peine s'énoncer comme tel dans la mesure où la catégorie reine de cette critique, le réel, est complètement indéterminée, et où la déterminer, c'est précisément réussir le défi que se propose Marx, aussi bien que de réussir là où Hegel a échoué.

Les différentes formes de critique se soutiennent les unes les autres dans le projet unique quoique sous-jacent de déterminer le véritable objet de la critique marxienne : le réel politique. La fonction critique consiste donc aussi bien à déstabiliser une théorie concurrente parce que ne prenant pas pour norme cet objet - en l'occurrence celle de Hegel - qu'à tenter par opposition et dans la confrontation à déterminer cet objet. La section sur le pouvoir législatif va nous permettre de préciser notre propos.

27. Le vocabulaire de Marx a cet égard est on ne peut plus clair quoiqu'il ne nous renseigne jamais avec précision sur le contenu déterminé de ce qui est appelé réel. En effet, dans le discours critique à l'égard de Hegel, c'est toujours la " vraie » sphère, " la vraie » réalité, ou les choses concrètes qui sont appelées en renfort, de même qu'il s'agira contre la monarchie dont les Principes de la philosophie du droit semblent spéculativement garantir la légitimité d'en appeler à la « vraie » démocratie, à travers une méthode qui repère précisément les vides et erreurs de la théorie hégélienne, en en expliquant la genèse, au nom de la « vraie » critique contre la critique « vulgaire ».

28. Op. cit., p. 94.

29. Id. 
Il s'agit du moment où Hegel s'intéresse à l'organisation du pouvoir législatif pour lui-même tel qu'il peut être mis en œuvre pour légiférer et faire appliquer les lois, dans le cadre de sa théorie plus globale sur la monarchie constitutionnelle comme État à même d'instituer la liberté et de dépasser la particularité de la société civile vers l'universel. Il s'agit donc d'une section très technique que Marx examine pourtant avec le plus grand soin, car quand bien même Hegel converge avec les idées de son temps, il offre cependant " une véritable théorie spéculative de la représentation » ${ }^{30}$ dans le droit fil de sa conception des rapports entre société civile et État, celle-là même que Marx n'a de cesse de critiquer.

Ce qui nous intéresse commence avec le paragraphe 302 dans lequel Hegel montre que les états doivent servir de médiation entre le peuple, luimême divisé en individus particuliers, et le gouvernement comme instance du pouvoir exécutif, et plus précisément le pouvoir du prince. Le but de Hegel est de réconcilier l'exécutif avec ceux sur lesquels il doit s'exercer afin qu'ils ne soient pas séparés absolument comme deux «extrêmes » ${ }^{31}$. La médiation permet justement à la société civile de ne pas être en dehors du politique, ce qu'elle est par essence sans cette médiation. La société civile, par les états, se nie en tant que particularité non politique pour avoir accès à la politisation et à l'universel. On comprend donc d'emblée pourquoi Marx porte son attention sur ce passage et les suivants : il s'agit de la section censée enrayer le non-politique de ce qui est par ailleurs attestée par l'expérience et la modernité : la fragmentation voire la dissolution du politique (aussi et surtout comme lieu de l'universel) dans les différences individuelles d'intérêts.

Ce que Marx pointe très rapidement ${ }^{32}$, c'est que rien ne permet à la théorie hégélienne de montrer qu'il s'agit effectivement d'une médiation, ou plutôt que si médiation il y a, la théorie de Hegel ne peut l'expliquer mais tout au plus se borner à la constater: " mais de quelle façon les états sont censés s'y prendre pour commencer à unifier en eux deux dispositions d'esprit contradictoires, cela n'est pas indiqué $\gg{ }^{33}$. Il faut bien comprendre la tonalité de ce passage : le problème n'est pas tant que Hegel a tort et travestit la réalité au profit de sa seule cohérence systématique, mais qu'il a peut-être raison sans que sa théorie puisse en rendre raison, ce qui est tout à fait autre chose. Hegel peut sans doute à juste titre montrer la nécessité d'une médiation, mais il se fourvoie dans la solution toute spéculative qu'il propose, ce qui nous permet de comprendre en creux que la théorie doit envisager un moyen de proposer une telle médiation, en s'installant au sein du réel lui-même et non du point de vue d'un système de philosophie.

30. F. Hegel, Les Principes de la philosophie du droit, trad. J.-F. Kervégan, Paris, Presses Universitaires de France, 1998, p. 501.

31. Ibid., p. 504.

32. Op. cit., « les états sont la contradiction posée de l'État et de la société civile bourgeoise au sein de l'État. Ils sont en même temps l'exigence de la résolution de cette contradiction », p. 160 .

33. Id. 
La critique se fait plus précise au paragraphe suivant et Marx a à cœur de déloger le problème que pose cette fausse médiation ${ }^{34}$ qui propose « la solution de l'énigme ${ }^{35} \gg$ consistant à dire que dans l'élément des états au sein du pouvoir législatif, l'état privé accède à une signification politique ; soit pour Marx, « la signification politique de l'état privé, de l'état non politique qui est l'élément des états, une contradiction in adjecto ${ }^{36}$. Hegel semble en effet se contredire en disant que les états et donc les états privés ont une signification politique en eux-mêmes, alors qu'ils émanent de la société civile : «l'état privé ressortit à l'essence de cet État, à sa politique. Par conséquent, ce dernier lui donne également une signification politique, c'està-dire une autre signification que sa signification effective $»^{37}$. Par un saut que rien ne semble justifier, Hegel, pour Marx, transforme la particularité des états en signification politique : "la différence entre les états de la société civile bourgeoise devient une différence politique $»$.

Mais la critique marxienne, dans le prolongement de ce qui vient d'être dit, prend une couleur nouvelle en ce que Marx en appelle à l'histoire pour déstabiliser la théorie de Hegel ; c'est l'histoire qui sert ici de critère, de norme pour mesurer le réel et son écart avec la théorie hégélienne. Puis Marx repart sur les contradictions internes au discours hégélien. L'explication historique vaut donc comme garant éventuel de la théorie hégélienne mais celle-ci est néanmoins sommée de se présenter comme cohérente. Autrement dit, Marx ne convoque pas la réalité historique comme critère du discours hégélien mais comme indication de son inconséquence, que les contradictions internes et théoriques viennent ensuite confirmer. Avant de remarquer que l'histoire est convoquée, il faut remarquer quelle fonction critique elle joue aussi ; or il s'agit bien de la même fonction que la réalité politique indéterminée : sa mention et son observation vérifient la théorie. On retrouve donc l'appel au réel comme norme invisible, insituable et indéterminée, à l'aune de laquelle il faut juger la pertinence et la cohérence du discours hégélien. Mais ici, il y a néanmoins un surcroît de détermination, puisqu'il ne s'agit plus d'intuitions ou d'observations qui seraient hors du texte, mais de connaissances historiques - le réel est bien là, mais à titre d'explication génétique. C'est parce que la société s'est réellement conduite de telle manière que ce qu'il s'agit de connaître politiquement est tel. L'histoire ne sert pas encore de base à la théorie comme il en sera le cas à partir de L'Idéologie allemande, l'histoire vérifie seulement la théorie.

C'est encore ce que l'on observe avec le passage suivant: "Ce n'était pas parce qu'ils participaient à la législation qu'ils étaient des états ayant une dimension politique, mais c'était au contraire parce que dans la mesure où

34. Op. cit., p. 165 : « il est important de suivre très précisément ce développement ».

35. Op. cit., p. 164.

36. Ibid.

37. Ibid. 
ils étaient des états ayant une dimension politique qu'ils participaient à la législation. Quel peut donc bien être le point commun avec l'état privé de Hegel, qui en tant qu'élément législatif, devient un morceau de bravoure politique, et accède à un état extatique, à une signification et à une efficience politique qui ont un charme particulier, frappent les esprits et sont tout à fait exceptionnelles ? ${ }^{38}$. Ce qui est intéressant ici et qui confirme ce qui vient d'être énoncé, ce n'est pas tant en effet une éventuelle erreur historique de la part de Hegel, mais plutôt une controverse souterraine sur la signification même du sens du politique. S'il y a méprise chez Hegel, c'est qu'il tente de faire de la participation des états à la législation une signification politique en soi ; or c'est ce que conteste Marx : s'il y a participation au pouvoir législatif, c'est que le politique est présupposé par cet acte non concrétisé à travers lui. Autrement dit, encore pour Marx, l'enquête doit se charger de comprendre ce qu'est précisément cette présupposition et non la reconduire facilement comme semble le faire Hegel. S'il y a travestissement du réel, ce n'est donc pas tant parce que Hegel justifierait rationnellement au sein de son système des éléments que personne ne peut constater mais plutôt l'inverse : sa théorie ne fait que redoubler le réel, sans l'expliquer et sans montrer en quoi et pourquoi celui-ci est comme il est. Ce rôle sera dévolu à l'histoire, mais ce n'est pas encore précisément ce qui est en jeu ici.

Enfin, la dénonciation de l'artificialité de la solution hégélienne qui intervient toujours dans le commentaire du même paragraphe achèvera de nous convaincre. Dans un premier temps, Marx délaisse la pensée critique de cette fausse solution qu'est la médiation pour s'intéresser à la caractérisation hégélienne de la société civile: "mais il ne s'agit pas ici d'un passage progressif, il s'agit au contraire d'une transsubstantiation, et il ne sert à rien de ne pas vouloir vivre ce fossé, dont l'existence est démontrée par le saut même qui nous permet de le franchir $»^{39}$ : il y a fossé, comme le dit Marx, et la médiation ne peut faire un pont entre l'une et l'autre réalité. Mais dans le droit fil des remarques précédentes, Marx semble plutôt critiquer la conception hégélienne du politique : s'il y a un tel écart, c'est qu'on ne comprend pas ce qui fait que l'éventuelle jointure avec l'État, fût-il universel, puisse être politique. D'où le report de la critique sur le concept même de société civile bourgeoise en insistant sur son manque d'organisation et sur le fait que Hegel a méconnu ses caractères réels. Marx remarque ainsi que les seules caractéristiques de cet état sont l'absence de possession et le travail immédiat et « concret». Ainsi, le concept de société civile est très instable et la théorie hégélienne a du mal à l'appréhender. Soit ses catégories transforment la réalité, soit elles ne peuvent l'expliquer, ce qui déporte son intérêt théorique sur le seul fait de rendre justice à la théorie elle-même, en l'occurrence au système circulaire hégélien.

38. Op. cit., p. 167.

39. Ibid., p. 197. 
C'est dans la suite de ce passage que s'affirme nettement l'opposition méthodologique et théorique entre Marx et Hegel. Marx s'attaque en effet proprement à la difficulté logique du concept de médiation entre société civile et État, soit le cœur du reproche théorique le plus fort à l'encontre de Hegel : «la médiation que Hegel veut ici accomplir est une exigence qu'il dérive non pas de l'essence du pouvoir législatif, de la détermination qui lui est propre, mais bien plutôt de la considération qu'il a pour une existence résidant hors de la détermination essentielle. C'est une construction issue de cette considération $»^{40}$. Hegel avance une telle thèse parce qu'il croit pouvoir élaborer une construction théorique uniquement à partir de ce qu'il observe. Or c'est précisément ce qui manque chez Marx : une saisie du réel qui ne serait pas simplement une observation dépourvue de méthode. À Hegel au contraire, on ne peut absolument pas faire le même reproche : puisque le réel est nécessairement toujours déjà théorique, puisqu'il est d'emblée chargé du sens que l'autodéploiement de l'esprit laisse sur son passage, il n'y a plus qu'à le recueillir dans une construction philosophique qui lui est théoriquement homogène. C'est pourquoi Marx, à la fin du paragraphe, attaque Hegel précisément sur ce terrain: «il s'ensuit à l'évidence que cet Etat n'est pas un vrai État, parce qu'en lui les déterminations étatiques (parmi lesquelles le pouvoir législatif) ne doivent pas être considérées en et pour soi, de façon théorique, mais au contraire de façon pratique, comme des puissances non pas autonomes mais grevées d'une opposition, non pas issues de la nature de la chose, mais régies par les règles de la convention ${ }^{41}$. Tout se passe comme si l'élément réel était la société civile, tandis que l'élément abstrait, purement théorique, serait l'État. Si donc Hegel n'a pas vraiment pu matérialiser au sein de sa théorie la médiation nécessaire entre ces deux sphères, il ne peut non plus penser adéquatement la société civile, c'est à dire l'embrasser dans sa théorie et la comprendre parfaitement ; aussi est-il obligé de la travestir en lui apposant un modèle conceptuel qui ne lui appartient pas, pas plus que la mesure systématique et philosophique dont il se sert pour l'évaluer: "il semble alors d'abord qu'ici, ni l'extrême de l'universalité empirique, ni le principe princier ou monarchique ne se confrontent à l'extrême de la singularité empirique ${ }^{42}$. Cette médiation et son absence, ainsi qu'on l'a dit, Marx les remarque mais n'en traite pas pour autant. Nous ne trouvons dans les pages qui suivent aucune étude de cette «singularité empirique ». Aussi se borne-t-il à une révision de cette théorie et à une constatation quasi-stérile de cette impuissance et incapacité théorique. De même, le prince devait assurer la médiation que Marx critique, mais c'est encore une fois le vide conceptuel de la notion de politique que Marx pointe pour fragiliser cette éventuelle médiation; le prince ne peut jouer ce rôle puisqu'il a lui-même besoin d'une médiation entre lui et la société civile

40. Ibid., p. 181.

41. Ibid., p. 180.

42. Ibid., p. 181. 
bourgeoise $^{43}$, n'étant pas d'emblée politique. Ces extrêmes sont comme Janus. On voit donc que le fonctionnement de la critique suit le schéma que nous avons tenté de mettre en évidence ${ }^{44}$.

Une autre séquence s'ouvre avec le commentaire du paragraphe 307 qui concerne le rôle de l'hérédité familiale dans la transmission du rôle de représentant parlementaire de l'état substantiel, et que Marx analyse comme la dépendance du pouvoir princier et du majorat vis-à-vis de la propriété privée. Ce que Hegel désigne comme le principe naturel de la famille, Marx en fait «l'abstraction effectuée de la propriété privée» pour mieux repérer par la suite « l'animalité » de la conception hégélienne : " c'est par la naissance que les plus hautes activités de l'État coïncident avec les individus, de même que la place de l'animal, son caractère, mode de vie, etc., lui sont immédiatement innés. L'État dans ses plus hautes fonctions reçoit une effectivité animale ». De quoi s'agit-il ici pour Marx ? Ses propos consistent à rendre étrangère la connexion qui semble si logique à Hegel entre la naissance individuelle et le pouvoir législatif, et c'est pourquoi il parle de $«$ miracle $^{45} »$; ce faisant, il exhibe a contrario la nature sociale des liens politiques au sens où ils sont les produits d'une certaine culture en même temps que d'une certaine histoire. Hegel rate donc la socialité du social et transforme un rapport social - ce qu'il faudrait examiner - en un rapport naturel au lieu d'enquêter sur le fait que «l'assentiment seul fait de la naissance de cet être humain la naissance d'un roi ; c'est donc l'assentiment, et non la naissance, qui fait le roi » ${ }^{46}$, et que «dans la noblesse, c'est donc naturellement la fierté du sang, de l'ascendance, en bref la biographie de son corps, c'est naturellement cette manière zoologique de voir les choses, qui possède dans l'héraldique la science qui lui correspond. Le secret de la noblesse est la zoologie ${ }^{47}$. Il s'agit d'un passage rhétorique qui consiste à renverser la logique et la prétention hégélienne : Hegel veut saisir la rationalité politique de la modernité mais n'en saisit qu'une forme tantôt abstraite tantôt passée, c'est-à-dire qu'il rate son objet dans tous les cas. Mais ce morceau de bravoure rhétorique permet à Marx de continuer de façon plus serrée en repérant deux moments: d'abord, il montre qu'en réalité, ce n'est pas tellement l'homme, l'individu dans sa matérialité ou corporéité qui importe, mais bien plutôt ce à quoi cette biologie est attachée, c'est-à-dire la propriété. L'animalité de la conception hégélienne n'était donc qu'un prélude à la véritable charge critique. Elle lui permet en effet de déduire dans un deuxième moment que la qualité politique du majorataire

\section{Ibid., p. 183.}

44. Une fois mises en perspective les insuffisances éventuelles et incohérences de la théorie hégélienne, la tension critique semble par ailleurs se relâcher puisque Marx enchaîne sur une page qui additionne les comparaisons acerbes pour ridiculiser la dialectique de Hegel et son incapacité à trouver une médiation véritable.

45. Ibid., p. 204.

46. Ibid., p. 205.

47. Ibid. 
ce n'est pas lui-même mais bien sa propriété, c'est-à-dire encore le droit. Commentant ces moments, il écrit « pour ce qui concerne le premier point, il s'ensuit que le majorataire est le serf de la propriété foncière et que dans les serfs qui lui sont assujettis, apparaît seulement la conséquence pratique du rapport théorique dans lequel lui-même se trouve avec la possession foncière ». À l'intérieur du discours hégélien, c'est une tout autre logique qui fonctionne et à son insu semble-t-il : la logique de la pensée bourgeoise, mais Marx ne l'appelle pas encore de cette manière ; "la profondeur de la subjectivité germanique apparaît partout comme la grossièreté d'une objectivité dépourvue d'esprit ».

Enfin, Marx en déduit une conséquence primordiale :

L'indépendance, l'autonomie dans l'État politique dont nous avons jusqu'à présent suivi la construction est la propriété privée qui en sa pointe apparait comme possession foncière inaliénable. C'est pourquoi l'indépendance politique ne découle pas ex proprio sinu de l'État politique, elle n'est pas un don de l'État politique à ses membres, elle n'est pas l'esprit qui l'anime, mais les membres de l'État politique reçoivent au contraire leur indépendance d'une essence qui n'est pas l'essence de l'État politique, d'une essence du droit privé abstrait, de la propriété privée abstraite. L'indépendance politique est un accident de la propriété privée, non la substance de l'État politique... la signification qu'a la propriété privée dans l'État politique est sa signification essentielle, vraie $^{48}$.

Cette dernière séquence exprime presque à elle seule à la fois la diversité des formes de critiques que Marx met en œuvre ainsi que l'unicité difficilement énonçable de leur objet. Diversité car on assiste à quatre moments : d'une part, Marx commence par souligner l'abstraction donc le lien diffus au réel que mobilise la théorie hégélienne pour mieux en fustiger immédiatement après la fausse matérialité qu'elle semble se conférer dans sa «zoologie ». Mais celle-ci n'est elle-même convoquée que pour être reconduite à une abstraction plus profonde et complexe : celle du droit, et en l'occurrence la propriété. En guise de profondeur théorique, Hegel est ramené à une banalité de classe tout à fait prosaïque : ici comme ailleurs, le concept est issu d'intérêts de classe bourgeois. On pourrait souligner la précocité d'une telle remarque critique, semblant annoncer les meilleurs développements conceptuels de l'Idéologie allemande (quoi de moins étonnant dans une pensée en cours d'élaboration?), mais Marx poursuit de toute façon pour atteindre «la signification de la propriété privée » et donc de l'État telle que le pense Hegel, signification qui est désormais « essentielle, vraie ${ }^{49}$ ». On retrouve ce vocabulaire normatif dont on a dit qu'il ne peut que pointer vers le sentiment qu'a Marx de toucher à son objet : il ramène le concept à sa banalité théorique, au droit - c'est à dire à ce qui se

48. Ibid., p. 206.

49. Ibid. 
trouve à l'interaction du social et de l'idéel - puis à la logique des intérêts bourgeois pour en déterminer la logique sous-jacente et vraie : la médiation n'est rien d'autre que l'expression juridique d'intérêts réels et pratiques.

Dans ce passage, il devient très difficile de discerner ce qui relève de la critique interne ou externe car Marx passe de l'une à l'autre (d'une abstraction mal dégrossie dans l'animalité «zoologique » à la signification "vraie» de la propriété privée) pour ne s'arrêter que là où il pense avoir trouvé ce qui détermine la non-pertinence du concept hégélien, en l'occurrence le réel d'une position de classe bien concrète. Comme on a tenté de le montrer plus haut, si l'on ne peut discerner la méthodologie critique avec certitude, c'est que celle-ci n'est pas le véritable enjeu, à l'inverse de cette "signification essentielle » qu'est la propriété bourgeoise. Mais ce point d'arrêt sert aussi d'indication méthodologique en ce qu'elle marque précisément ce que la théorie doit investir ; faute d'une élaboration tout à fait claire, c'est la norme de la vérité "essentielle» qui sert ici de référent. Autrement dit, la fonction critique bouge faute de saisir complètement son objet, et c'est le droit qui a la priorité ici parce qu'il semble vraiment émaner de ce réel qu'il s'agit de circonscrire.

\section{La tentative de lecture rétrospective : une critique de la lecture de ce texte}

Si l'on suppose réglé le problème de savoir quel peut être l'objet de la critique marxienne, on peut maintenant aller plus loin en comprenant que le choix du concept de critique est seul apte à nous faire comprendre la rédaction de ce texte bien connu des commentateurs. Tout comme l'Idéologie allemande plus tard, il s'agit d'une œuvre de jeunesse mais surtout destinée uniquement à son auteur dans la mesure où sa rédaction même sert d'élucidation méthodologique. Or les commentateurs ont tendance à le comprendre du point de vue exclusif de la maturité méthodologique, ce qui les conduit à localiser des thèses bien précises, toujours intéressantes, mais en occultant souvent la progression méthodologique dont le texte porte les traces disparates.

L'analyse et la circonscription de la thèse du texte ne sont pas faciles dès lors que l'on décide de maintenir l'idée d'un travail «pour soi », c'est-àdire d'un travail qui ne se propose pas tant de constituer une théorie pour elle-même que d'être la base d'une réflexion personnelle pour l'auteur. Or c'est le concept de critique qui est seul en mesure de nous rendre attentifs à l'aspect inchoatif plutôt que thétique de cette œuvre car dans son indétermination même et ses thématisations disparates au cours du texte, il nous met sur la voie d'une méthode et d'une conceptualité se cherchant elle-même. Comme on a essayé de le montrer plus haut, retenir de ce texte qu'il s'agit d'une inversion de la thèse hégélienne sur l'État, c'est en exhiber l'analyse la plus saillante tout en passant sous silence les errements laborieux et multiples du discours. À l'inverse, en postulant l'unicité d'un 
objet critique que la fonction critique doit dégager au contact de la thèse hégélienne, une attention nouvelle est permise sans qu'elle puisse mettre au jour une thèse aussi frontale que celle que l'on décrit habituellement.

Mais si Marx, en réalité, ne sait pas encore ce qu'il cherche - d'où l'inévitable explication avec soi et aussi avec son propre héritage : Hegel -, si ce but n'a jamais été ni formulé ni a fortiori thématisé par l'auteur luimême, on ne peut qu'essayer de deviner en se confrontant en quelque sorte à une lecture génétique et philosophique de ce premier travail philosophique, lecture qui dès lors ne peut jamais totalement faire l'économie d'une vision rétrospective et conciliatrice. Toutefois, l'attention au concept de critique permet de ne pas absorber tous les enjeux et détails du texte dans une simple rétrospection, soit une fois pour toutes dirigée vers le Marx de la maturité, soit, dans l'interprétation rigoureusement opposée, celle d'un texte développant une thèse singulière et se suffisant à elle-même, à l'extérieur pour ainsi dire du corpus marxien connu.

C'est ce qui ressort semble-t-il de la lecture de M. Abensour qui voit dans ce texte la découverte du véritable lieu du politique. Il s'agit pour lui de replacer Marx dans la philosophie politique et notamment dans sa face cachée: derrière l'idéal politique libéral se cacherait en effet une réactivation humaniste de la politique (dont les origines remonteraient au moins à Machiavel) qui tend à faire du politique un lieu autonome, dans le prolongement d'une anthropologie humaniste en fait héritée des Grecs et d'Aristote, qui veut que l'homme soit un animal politique ${ }^{50}$. D'où les nombreux appels - majoritairement suggestifs dans le texte - à la « vraie démocratie " comme seul régime à même d'investir à fond ce lieu du politique que Marx découvre. Lecture en effet séduisante mais qui a le défaut de verser d'une certaine manière dans la surinterprétation, adossée à la vision complète de l'œuvre marxienne ultérieure et des nombreux thèmes qui y seront développés, en faisant de ces remarques disparates un tout cohérent, développé et thétique - une nouvelle théorie au bord de l'achèvement. Le meilleur contre-argument à une telle thèse est le fait qu'en réalité, Marx dit très peu de choses sur ce qu'il appelle plusieurs fois la "vraie démocratie », à l'instar d'ailleurs du Traité Politique de Spinoza, qu'il avait lu un peu avant selon toute vraisemblance. Il nous semble que c'est là précisément ne pas tenir compte ou pas assez de la dimension agonistique c'est-à-dire proprement dynamique du texte, qui, même définie minimalement, permet pourtant de mettre l'accent sur la confrontation d'une pensée qui n'est pas encore une théorie complète. L'effort doit donc se porter sur la pertinence et sur le but de cette confrontation, non simplement sur ce que l'on peut théoriser à partir d'elle et au fond sans elle. À l'inverse, on est conduit dans le commentaire de $\mathrm{M}$. Abensour quasi immédiatement à faire de la critique « une herméneutique émancipatoire ${ }^{51}$ ». Plutôt qu'une philosophie critique contre Hegel et à la recherche de la matérialité,

50. M. Abensour, La Démocratie contre l'État, Paris, Le Félin, 2012, p. 27.

51. Id., p. 116. 
M. Abensour veut voir une philosophie politique dirigée vers l'expérience de la liberté humaine et moderne telle que manifestée dans le mouvement révolutionnaire ${ }^{52}$, ce qui conduit à penser la société civile bourgeoise comme le lieu individuel et égoïste qui épuise le social sans être immédiatement politique. Une telle émancipation voudrait donc politiser le social de sorte qu'il ne s'agisse pas seulement d'un état de nature économique. L'émancipation passerait donc par la reconduction du politique au social ${ }^{53}$. Penser l'essence du politique au regard du sujet réel qu'est le démos et non plus comme le fait Hegel au regard de l'idée, ce que proposerait Marx ici, constitue un dévoilement de la fonction propre du politique selon son véritable sujet comme le montrent, du reste, pour Abensour les autres textes marxiens de la même période et sa correspondance avec Ruge: la préoccupation de Marx est de dégager l'essence du politique, le plus précisément possible.

L'idée logique hégélienne fonctionne pareillement qu'il s'agisse de l'État ou de la nature, en sorte que pour Marx lu par M. Abensour, la philosophie du droit de Hegel n'est que le faux-semblant d'un savoir réel du politique ${ }^{54}$. À l'inverse, dans le sillage de Spinoza, l'essence de la politique pour Marx ne peut se ramener à la seule opposition maître-esclave. L'élément politique est un lien spécifique, irréductible à une dialectique des besoins ou à une dérivation de la division du travail, " comme un moment dont une société humaine vouée à la liberté ne peut faire l'économie, sous peine de retomber dans le monde animal politique (vivre et se multiplier) $\gg^{55}$. Si l'on pense la politique seulement en termes de domination, alors effectivement la monarchie en est l'apothéose, ou alors la visée de la politique est l'instauration d'une communauté entre les hommes. Marx, en ce sens, veut la disparition de l'État pour l'avènement de la vraie démocratie. La démocratie dévoile l'essence du politique, «l'homme socialisé ${ }^{56}$. Hegel, en bon moderne, fait de l'homme l'État subjectivé dans la personne du prince, et il s'agit pour Marx de montrer qu'à l'inverse, dans la démocratie, l'homme comme être générique, le démos parvient dans l'État et par l'État à la subjectivation. C'est parce que l'homme se donne une constitution qu'il se révèle homme socialisé, zôon politikon. C'est seulement dans la sphère politique que le membre de la société signifie homme.

Mais une telle interprétation suppose le concept d'un sujet politisé en l'espèce du peuple, prêt à se doter lui-même d'un régime politique adéquat ${ }^{57}$. De même, si l'on a pu soutenir une lecture rigoureusement inverse ${ }^{58}$, c'est

52. Ibid., p. 23.

53. Ibid., p. 127.

54. Ibid., p. 134.

55. Id.

56. Ibid., p. 150.

57. Ibid., p. 197.

58. Par exemple, pour A. Grandjean, pour qui il s'agit essentiellement d'une critique de la politique et non du social. Pour lui l'objet de la critique est la politique en tant que lieu 
sans doute aussi faute d'une vigilance à l'égard de ce qu'est et de ce que fait le discours critique ici. Ainsi, on subordonne a priori le texte à un destin herméneutique (il s'agit d'une étape vers la conceptualisation de l'aliénation des Manuscrits de 1844 par exemple), ce qui autorise toutes les interprétations pourvu qu'elles se rattachent plus ou moins bien à l'objectif final et qu'elles donnent une image plus ou moins fidèle du chemin qui y parvient. Que faire ainsi des différentes formes de critique dont on a voulu rendre raison? L'interprétation de $\mathrm{M}$. Abensour nous oblige à les passer sous silence ou à les englober dans une tentative incompréhensible car indéterminée de détruire pour Marx l'héritage théorique de Hegel sans que celle-ci ait pu aboutir car Marx n'a jamais publié son texte.

Plus précisément, pour A. Grandjean pour prendre un autre exemple, «la vraie critique» que Marx appelle de ses vœux et dont il semble se considérer comme un représentant relève d'une généalogie des contradictions et non d'une simple exposition de celles-ci. Il remarque que selon Marx déjà la communauté réelle est une communauté de lutte ${ }^{59}$. On constate sans peine pour lui une fluidité totale de la société civile bourgeoise qui fait éclater toute communauté stable. Pour séduisante que cette interprétation puisse paraître, elle consiste également à isoler un moment du texte pour en faire le porteur de la signification critique de la totalité de l'ouvrage et donc à escamoter la dynamique de la fonction critique du texte. Certes, il s'agit bien du seul moment réflexif où Marx revient sur son propre discours pour en préciser la détermination et le sens critique, mais il ne faut pas oublier la progression même de la révision critique qui semble indiquer qu'il n'était pas en possession d'un tel sens avant la rédaction de l'ouvrage. Il semble que celui-ci se fait donc au contact du texte hégélien. C'est pourquoi il nous faut maintenant tenter de comprendre pourquoi précisément le choix d'une confrontation théorique s'est porté sur Hegel. Il s'agit d'une question mise de côté jusqu'à présent et la seule réponse que l'on puisse y donner au terme de notre parcours est que l'héritage hégélien a été aux yeux de Marx particulièrement fécond dans l'élaboration progressive de sa critique.

La rédaction de ce texte intervient dans un contexte historicophilosophique tout à fait particulier. L'année 1843 est en effet une année décisive pour la formation intellectuelle du jeune Marx ainsi que pour ses aspirations. Lorsque Frédéric Guillaume IV accède au trône, un certain infléchissement libéral vers plus de libertés et moins de censures, notamment pour la presse et l'intelligentsia, a pu être envisagé et espéré, mais l'année 1843 enterre ces espoirs. C'est ce qui conduit sans doute Bruno Bauer, le comparse jeune-hégélien de Marx à publier La Montée et la chute du

moderne de l'aliénation, et la méthode critique marxienne est ici une dialectique sans médiation ; cf. «La dialectique sans la médiation, le jeune Marx et l'abîme qui sépare le social du politique », Presses Universitaires de France, Les Études philosophiques, 2008/4 $\mathrm{n}^{\circ} 87$, p. 539 à 554 .

59. Id., p. 544. 
radicalisme allemand de l'année 1842. La censure est rétablie par le monarque sur tous les ouvrages et la presse. Le 21 janvier 1843, suite notamment aux articles de Marx sur le vol de bois, la Gazette Rhénane est interdite. C'est donc face à une situation politique qui s'envenime de plus en plus que les positions intellectuelles des Hégéliens se crispent et se divisent en mouvances antagonistes. Face à ces difficultés, l'opposition avec le gouvernement ne peut plus prendre la forme que d'une spéculation dénuée de toute pratique, dans le sillage d'une certaine interprétation de Hegel, et c'est effectivement ce que choisissent Ruge, Feuerbach et Bauer notamment. L'idée est que la philosophie, en particulier spéculative, ne peut plus se borner à recueillir passivement le sens du réel mais doit au contraire porter au jour la véritable nature despotique du pouvoir prussien, autant que de n'importe quel régime politique d'ailleurs, tant qu'il s'agit d'un travail essentiellement théorique. Ruge choisit plutôt une forme de "pessimisme critique » qui vise à spéculer sur le réel et la politique mais dans une optique qui se veut alors critique, sans plus d'action politique que l'option précédente. Enfin, Marx adopte sans doute une voie médiane, entre spéculation théorique et critique qui doit s'articuler à une forme d'action politique, fût-elle pour l'instant indéterminée ${ }^{60}$.

Ce qui est décisif selon Kouvélakis par exemple, c'est le passage du Marx démocrate au Marx révolutionnaire dont la Critique de la philosophie du droit de Hegel serait le témoin, ou l'élaboration théorique initiale. Mais il nous fournit des éléments de réponse quant au choix de Hegel en remarquant qu'avant de le jeter sur les chemins de l'exil, la crise de 1843 se traduit par un geste qui peut sembler étrange pour ce partisan fervent de la « critique dans la mêlée » mais qui se révèle assez caractéristique de ces moments de basculement historique : la retraite dans la solitude du cabinet d'études. C'est, pour l'essentiel, dans celui de son beau-père, à Kreuznach, que Marx fera ses adieux intellectuels à son passé philosophique et, comme on pouvait s'y attendre, avec sa formation philosophique initiale: Hegel, qui est aussi une confrontation avec le bilan de la Révolution française. Mais l'étrangeté de ce contexte historico-philosophique disparaît si l'on pense que l'antagonisme entre un événement historique et l'éventuel accueil dudit événement dans la spéculation philosophique ne constitue pas ce qui est sous-jacent au texte même et qui pourrait ressurgir de façon connexe ou souterraine, mais bien le problème que la confrontation avec Hegel espère résoudre ou du moins débrouiller. Si en effet la philosophie théorique la plus accomplie n'aboutit qu'à un conservatisme stérile, et si d'autre part l'action politique doit être mise en branle, comment concilier les deux ? Si tant est que cela soit possible. Plus précisément, que faire d'une théorie qui n'aboutit pas à l'action alors même que celle-ci semble être appelée par l'histoire et

60. Selon Kouvélakis dont on se reportera à l'article pour une présentation historique plus détaillée ; cf. Eustache Kouvélakis, «Dans la quiétude de Kreuznach: Marx lecteur des Principes de la Philosophie du Droit de Hegel», Revue numérique Philopsis: http://www.philopsis.fr/spip.php?article322. 
les peuples comme ce fut le cas avec la Révolution française ? Un tel fossé requiert sans doute un examen méta-théorique à même de déterminer ce qui, dans la théorie, est inapte à connaître la situation présente et ainsi à la faire changer. Nous tenons que cet examen reçoit le nom de critique pour le Marx de cet ouvrage, mais comme l'examen lui-même doit fournir les éléments théoriques permettant de comprendre et le problème et la solution, celui-ci n'est absolument pas déterminé et se cherche donc au contact du texte hégélien, à la fois théorie spéculative la plus aboutie, la plus achevée et totalisante mais aussi la plus stérile et semble-t-il conservatrice. D'où alors ce que Marx appelle « critique dans la mêlée » ${ }^{61}$.

Finalement, tentons de répondre aux questions que nous nous étions posées. Il y a bien une unité de la critique marxienne à l'œuvre dans cet ouvrage mais il ne s'agit ni d'une unité systémique, ni d'une unité méthodique ou objective : il paraît singulièrement imprécis sinon impossible de définir ce que veut critiquer Marx ici, puisque le texte ne dissipe jamais unilatéralement les soupçons que nous avons soulevés. Impossible de montrer que Marx veut seulement s'en prendre à la théorie hégélienne, qu'il veut élaborer une théorie politique, ou seulement penser pour luimême. Mais précisément, cette enquête nous renseigne peut-être sur l'élucidation de cette notion "pour soi-même » qui paraît, à la lumière de cet ouvrage du jeune Marx, plus étrange qu'elle ne semble à première vue. Le véritable objet de la critique, au sens de la fonction critique, serait d'apprendre à connaître le social, le politique ou le réel. Le véritable objet consisterait donc dans la tentative pour l'instant encore balbutiante d'en appeler au réel dans sa dimension politique et sociale, non seulement en tant qu'il s'agit de le connaître mais aussi et surtout en tant qu'il représente le critère-étalon de la théorie, comme certaines récriminations de Marx dans ce texte nous l'ont montré. Le réel se voit donc affublé de deux déterminations différentes et qui n'apparaissent pas tout à fait circulaires : la théorie doit trouver un moyen de le connaître, mais c'est bien le réel lui-même qui jugera sur pièce la théorie. Or comment en faire à la fois un objet et un critère ? Dans une telle enquête, le réel risque d'être juge, partie et enquêteur.

C'est que nous avons encore affaire au jeune Marx ici, et selon P. Macherey dans l'article «Marx et la réalisation de la philosophie ${ }^{62}$ », s'il y a une chose que Marx n'a ni inventée, ni reprise de Hegel ou de l'économie politique, c'est l'entreprise critique qui était celle que s'étaient fixée les jeune-hégéliens ${ }^{63}$, entreprise qui considérait que Hegel n'était pas un accomplissement définitif de la philosophie mais plutôt un point de départ pour une pensée nouvelle. La caractéristique de la pensée de Hegel pour ses continuateurs est d'avoir voulu penser la pratique dans un cadre que la conscience épistémique produisait elle-même en s'autojustifiant, sans

61. Lettres de Marx à Ruge, in Critique de la philosophie du droit de Hegel, éd. Sociales, Paris, 2018, p. 299-309, citation p. 303.

62. P. Macherey, « Marx et la réalisation de la philosophie », Actuel Marx, Paris, 2005.

63. Ibid., p. 127. 
pourtant achever complètement cette entreprise puisque le cadre conceptuel qui devait s'appliquer à la pratique restait piégé dans la spéculation et l'idéel systémique de la pensée de $\mathrm{Hegel}^{64}$. C'est pourquoi la tentative jeunehégélienne était vite tombée dans les travers qu'elle dénonçait en continuant de vouloir faire rentrer comme de force la réalité dans la pensée, et non en la pensant pour elle-même, ce qui n'a pu que décevoir Marx. Si l'on veut se donner les moyens de dépasser la spéculation pour atteindre le réel, il faut s'y fondre, autrement dit agir, et la spéculation cède la place à l'action. Par l'intermédiaire de Hess qui avait mis l'échange au cœur de son anthropologie, Marx sera conduit à formuler une philosophie de la praxis qui devait aboutir au Capital ${ }^{65}$. Mais c'est bien l'ouvrage de Engels, La Situation de la classe laborieuse en Angleterre qui fournit la base sociologique indispensable à la théorie marxienne ultérieure, notamment pour l'élaboration de la conceptualité du Capital et l'élucidation de cette réalité étrange qu'est le capital.

Mais pour ce faire, il fallait encore se déprendre de l'objet à critiquer auquel les jeunes-hégéliens accordaient la part la plus importante de leur attention, c'est-à-dire la religion ${ }^{66}$. C'est donc sous l'effet de ce contexte théorique très particulier que les recommandations d'Engels (de lire les économistes, notamment anglais) vont trouver leur champ d'élection et conduire ainsi non plus à une critique de la religion ou à une anthropologie qui en découlerait mais, fort de nombreuses années de recherche en la matière, à une "critique de l'économie politique ». Or, comme le montre Macherey ${ }^{67}$, c'est notre ouvrage qui sert de charnière en montrant que la vérité de l'État repose sur la société civile plutôt que l'inverse. L'attention de Marx s'est donc déplacée vers la matérialité du social et la nécessité d'étudier les apports économiques qui président aux échanges et à la société pour être capable de la comprendre et ainsi éventuellement agir sur elle, dans des modalités qui sont encore toutes à définir. Si l'on suit cette reconstitution généalogique de la trajectoire intellectuelle marxienne, la séquence paraît claire : Marx rompt avec Hegel dans le sillage des jeunes-hégéliens sur fond d'une philosophie qui se veut pratique, puis rompt avec ces derniers au motif de l'échec de leur propre entreprise, et enfin rencontre Engels et se lance dans une toute nouvelle théorie qui s'alimente de l'économie politique, pour vouloir la critiquer et enfin agir dans ou sur la société. Nous avons voulu montrer que pour décisive que soit cette étape, elle est laborieuse et

64. Ibid., p. 128 : «Mais, dans la présentation que Hegel en a donnée, la pratique, ainsi mise particulièrement en valeur dans le cadre du devenir de la pensée, reste en quelque sorte engluée dans la théorie rationnelle à la dynamique de laquelle elle sert d'auxiliaire ; elle reste une pratique de théorie, vue au point de vue de la théorie: c'est pourquoi la tâche des successeurs de Hegel serait de dégager cette pratique de la théorie à laquelle elle est, chez lui, étroitement associée, et de lui accorder le rôle prééminent qui lui revient dans le destin du monde, ce qui est le programme propre à une philosophie de l'action ».

65. Ibid., p. 135.

66. Ibid., p. 140.

67. Ibid., p. 142. 
complexe dans la mesure où Marx sait pour lui-même qu'une théorie qui s'engage à comprendre le social et le politique doit s'atteler à enclore le réel en elle, à le comprendre mais sans le travestir, sans que la spéculation ou la métaphysique écrase ce réel. Cette élaboration difficile est celle d'une fonction critique qui se transforme au contact de son objet autant qu'elle tente de le saisir avec des outils théoriques inadéquats. Au moment de la rédaction, le choix d'affronter la théorie hégélienne de la société est le meilleur et le pire. Le pire, car avant de pouvoir y puiser des outils pour comprendre théoriquement le réel politique et social, il faudra la débarrasser de ses scories spéculatives et métaphysiques. Le meilleur, car il s'agit de s'expliquer avec un héritage qui revendique être dépositaire d'une tâche critique à travers Hegel. Toutes les conditions sont donc bien réunies pour qu'un affranchissement théorique puisse éclore à l'occasion de l'écriture d'un texte critique contre Hegel, mais cette éclosion est aussi celle d'une nouvelle théorie, que l'on ne peut déceler que si l'on s'attarde sur les mésaventures de la fonction critique. Il n'y a donc pas à s'étonner que l'objet de la critique soit finalement si peu déterminé ; sans être creux, il paraît en effet bien vide puisque les nombreux feuillets de Marx n'en disent pratiquement rien. Et pour cause, ce vide de la détermination ne pourra sans doute être comblé qu'avec le bon point de départ : l'étude des concepts de l'économie politique, qui sont censés décrire eux-mêmes les échanges, soit la base des interactions individuelles. Mais Marx n'a pas encore rencontré Engels. 